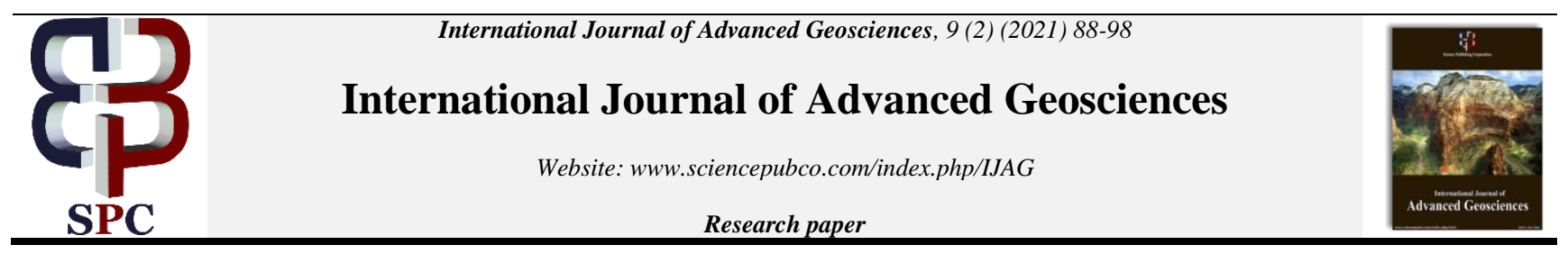

\title{
Machine learning approach to hydrocarbon zone prediction from seismic attributes over "GEM" field, Niger-Delta, Nigeria
}

\author{
Fredrick O. Oshogbunu ${ }^{1}$, Pius A. Enikanselu ${ }^{1}$ \\ ${ }^{1}$ Department of Applied Geophysics, Federal University of Technology, Akure, Ondo State, Nigeria \\ *Corresponding author E-mail: fredrickoshogbunu@gmail.com
}

\begin{abstract}
A computer programme (in Python language) was developed for the generation and performance assessment of predictive models, capable of combining relevant seismic attributes for reliable hydrocarbon zone prediction ahead of drilling. It attempts to resolve the problem of making accurate and efficient interpretations from a large database of derived seismic attributes. The research utilized post-stack 3D seismic volume for the delineation of structures and the generation of seismic attributes. Six faults were identified across the mapped horizons. Five seismic attributes were generated and exported from 3D seismic data as numerical values for machine learning analysis. The binary cross-entropy classification metric was used to evaluate the performance of the developed predictive models while an individual seismic attribute (Maximum Amplitude and Extract value) map was used to validate the predictive models. A correlation of well depth-to-top of selected horizons with the seismic depth slice surface was used for further model validation. The Multi-Layer Perceptron (MLP) model results enhanced the visibility of the other five hydrocarbon prediction zones. The MLP predictive model map gave higher precision of the predicted hydrocarbon zones over the Self-Organising Map (SOM) predictive model, thus reinforcing the confidence level of the former.
\end{abstract}

Keywords: Artificial Neural Network; Computer Science; Geophysics; Hydrocarbon Zone Predictions; Machine Learning; Seismic Attribute.

\section{Introduction}

Today's interpretation environment consists of various datasets such as seismic data acquisition large 3Ds with numerous processing versions, numerous wells with associated data, and a large database of seismic attributes. These could result in a large amount of data in different sizes. This, however, poses difficulties in making accurate and efficient interpretations by geoscientists (Sacrey and Roden, 2018). Moreover, interpretation based on a single generated seismic attribute will not often image directly and can be difficult to delineate optimal hydrocarbon presence in a reservoir (Chehrazi et al, 2013). Thus, there is the need to combine relevant seismic attributes for predicting optimal hydrocarbon zones. The machine learning techniques provide a modern veritable tool, through the writing of python codes.

Over the past eight years, the evolution of machine learning in the form of unsupervised and supervised neural networks has been useful to improve and gain more insights from seismic interpretation processes (Smith and Taner, 2010; Roden et al., 2015; Santogrossi, 2017; Roden and Chen, 2017; Roden et al., 2017). These forms of neural networks have been developed for the prediction of several reservoir characteristics using seismic attributes or well log datasets. Although, Roden et al. (2015) applied unsupervised neural networks, SelfOrganising Map (SOM) as regards the combination of related seismic attributes in defining stratigraphy, seismic facies, direct hydrocarbon indicator, and aspects of shale plays, such as fault/fracture trends and sweet spots. In general, unsupervised classification techniques are merely driven by the input data, whereas supervised classification techniques include external control provided and defined as data labels by the interpreter. These techniques use computer algorithms that iteratively learn from the data and independently adapt to produce predictive and reliable results. To assess the confidence level of the prediction, a validation of the results is critical in either case (Zhao et al., 2017).

For the machine learning analysis, five seismic attributes such as RMS amplitude, Maximum Amplitude, Average Energy, Average magnitude and, Extract value will be generated, selected, and exported from the 3D seismic cube as numerical values. They are well-known for revealing geologic features, relationships, and patterns in the seismic data that otherwise might not be noticed (Chopra and Marfurt, 2007). However, these were selected based on their measured seismic property called amplitude as well as their interpretative usage of directly indicating hydrocarbon in the subsurface.

\section{Theory: artificial neural network (ANN)}

A neural network, also known as an Artificial Neural Network (ANN), is a powerful computational model inspired by how the biological neurons work in the brain (Haykin, 2009; Shalev-Shwartz and Ben-David, 2014). The systematic approach adopted by the Artificial Neural 


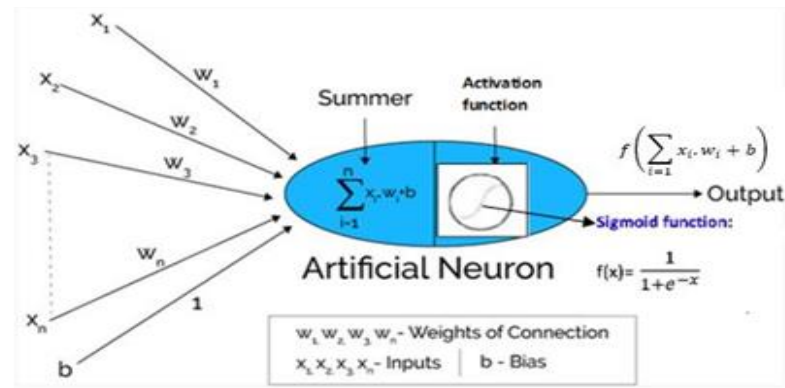

Fig. 1: Mathematical Model of Artificial Neural Network (ANN) Which Shows How It Works. Source: (Modified After Singh Gill, 2019). Note: (X_N) Number of Input (W_N) - Number of Weights of the Connection.

Network (ANN) may be described in few steps. First and foremost, when the input node $\left(\mathrm{X}_{\mathrm{n}}\right)$ of the neural network collects information from an external system in the form of patterns and images (Figure 1). Secondly, each weight of connection ( $\mathrm{W}_{\mathrm{n}}$ ) can be any real number (positive, negative, whole, or decimal number) that is moved from input nodes to the adjacent layer nodes. This is, however, multiplied by the corresponding input node $\left(\mathrm{X}_{\mathrm{n}}\right)$. Thirdly, the summer unit, sum up each multiplied weight of connection $\left(\mathrm{W}_{\mathrm{n}}\right)$ and corresponding input node $\left(\mathrm{X}_{\mathrm{n}}\right)$ in the processing unit. Then, the result is transferred to the activation unit where it is fired up by an activation function which could be linear or non - linear in nature, to rescale the multiplied sum as seen in Figure 1 (Singh Gill, 2019; Willems, 2019). Even though they are various activation function used in a neural network, the sigmoid function is a mathematical function that results in an "S" shaped curve, it is one of the most used activation functions which is categorized as a non-linear function (Singh Gill, 2019). Furthermore, a constant bias node or threshold (b) is applied in this process to maintain and control the connection between the input and the processor (Kumar and Mandal, 2018). This is, however, an additional parameter that is permanently set to 1 . The bias value is critical as a result of shifting the activation function to the left or right, which can make or determine the learning success (Singh Gill, 2019). Finally, a decisional output is expected from this operation at the output node (Figure 1).

In the entire process, the weights allocated to each input unit play a crucial role, as the stronger the weight is, the better would be the connection between the input and the processor. The decisional output obtained from the network is critically inspected. If the response output is found to be different than that of the expected output, the internal parameters like inputs, weights (or known as synaptic weights), etc., are rechecked and rescaled to establish a better match between the obtained and targeted outputs (Kumar and Mandal, 2018).

A neural network could comprise three or more layers: an input layer, one or many hidden layers, and an output layer. Data is received through the input layer, then the data is altered in the hidden layer and the output layers based on the weights applied to these nodes (Hurwitz and Kirsch, 2018). A neural network is said to be fully connected if every node in each layer of the network is connected to every other node in the adjacent forward layer (Cherazi et al., 2013). Although, learning with neural networks was proposed in the mid-20th century. It produces an effective learning paradigm and has lately been shown to achieve cutting-edge performance on numerous learning tasks (Shalev-Shwartz and Ben-David, 2014).

\subsection{Self - organizing map neural network}

Self-organizing Maps (SOM) is regarded as the most widely used clustering technique which is based on topological relations, the data is reorganized and projected onto a 2D manifold called the latent space (Kohonen 1982; Zhao et al., 2015; Zhao et al., 2016). It is categorized as an unsupervised machine learning technique. Architecturally, the SOM has two layers, the input layer and the output layer, which form a feed-forward structure from the (input) data space to the (output) feature space (Figure 2). The input layer provides different input seismic attribute patterns to the network, from which the network learns and then generalizes. On the other hand, the output layer comprises neurons (or nodes) that are usually spaced and arranged in a rectangular or square, or hexagonal grid shape. Both layers are fully connected and each connection is given an adjustable weight. However, this weight of adjustment is determined only by the difference between the input seismic attribute patterns and the nodes or neuron which is closest to it (the winner). For instance, when an input vector (seismic attribute value) is introduced to the network or the SOM allocates initial values to the neurons, the competition among nodes or neurons positioned on a discrete lattice is enforced. The winner of the competition is the node's weight at the output layer whose reference vector is the nearest to the input vector (Al Moqbel and Wang, 2011). In other words, the node with a weight vector closest to the input vector is the winner of the competition or tagged as Best Matching Unit (BMU) among its neighbouring neurons or nodes (Figure 2). Though, there are several ways to determine the shortest distance from each node's weight vector to the sample/ input vector, by running through all weight vectors, however, the most commonly used method is to calculate the Euclidean Distance between node's weight vector and the input vector (Ralhan, 2018). After several iterations for each sample in the training set, thus completing one epoch of SOM learning. The extent of neuron movement during an epoch is an indicator of the level of SOM learning during that epoch. The next epoch is undertaken by making adjustments to the learning parameters if an additional epoch is worthwhile. The process is complete when learning becomes insignificant (Roden et al., 2015). This results in an ordered network in which nearby nodes/ neurons will share certain similarities. Hence, the input of similar seismic attribute patterns does activate similar areas in the SOM model, creating local clusters/ grouping of nodes or neurons in the global self-organized network (Burguillo and Dorronsoro, 2013) as shown in Figure 2.

Mathematically, the below architecture of the SOM neural network is expressed as follows:

- $\mathrm{W}_{\mathrm{ij}}-\mathrm{Weight}$ vector or synaptic weight (this is defined as a connection from the ith component of the input vector to the jth neuron).

- $\quad \mathrm{i}$ - ith component of the input vector

- $\quad \mathrm{j}-\mathrm{jth}$ neuron or node of the output layer

- $\quad \mathrm{XY}$ coordinate - indicates the spatial location or position of each node in the 2D lattice

- Dimension $X$ - Total length of output nodes at $X$ coordinate axis

- Dimension $\mathrm{Y}$ - Total length of output nodes at $\mathrm{Y}$ coordinate axis 


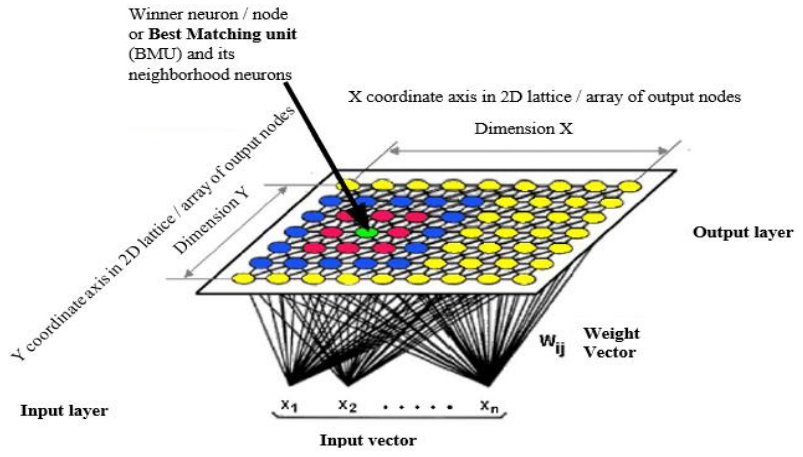

Fig. 2: Architecture and Mathematical Model of SOM Neural Network. This Is Showing Its Input Vector, Winner Neuron, Or Best Matching Unit and Its Neighborhood Neurons or Nodes. Source: (Modified After Burguillo and Dorronsoro, 2013).

\subsection{Multi-layer perceptron neural network}

A Multi-Layer Perceptron (MLP) is a feedforward artificial neural network model. MLPs are the simplest and therefore most commonly, used neural network architectures programs. This is due to their structural flexibility and good representational capabilities with a large number of programmable algorithms (Payandeh et al., 2009). It is categorized as a supervised machine learning technique. Architecturally, it comprises three layers, an input layer that contains several input nodes, an output layer that contains one or several output nodes, and at least one hidden layer which contains several nodes (Figure 3) or more than three layers (Hou et al., 2008).

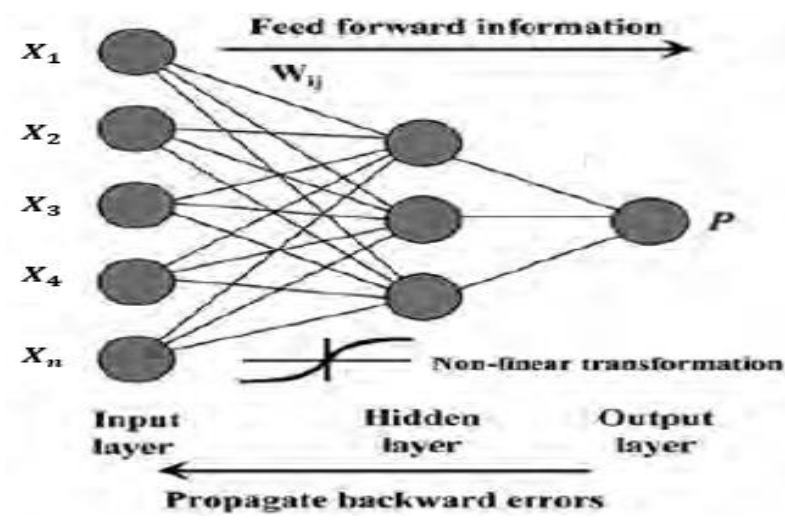

Fig. 3: Architecture of Multi-Layer Perceptron (MLP). Source: (Modified After Hou Et Al.,2008). The Input Data Fed into the Network System Flows Between These Layers and Performs the Operation.

Mathematically, the above architecture of MLP neural network is expressed as follows:

- $\mathrm{X}_{1}, \mathrm{X}_{2}, \mathrm{X}_{3}, \mathrm{X}_{4}, \mathrm{X}_{\mathrm{n}}-$ Seismic attribute inputs (Average Energy, Average Magnitude, Extract value, Maximum Amplitude and RMS Amplitude)

- $\mathrm{W}_{\mathrm{ji}}$ - weight of the connection

- $\quad \mathrm{i}$ - ith of the processing element (PE) in the input layer

- $\quad \mathrm{j}-\mathrm{jth}$ of processing element (PE) in the hidden layer and output layers

- $\left(\mathrm{X}_{\mathrm{n}}\right)$-number of seismic attribute input

- $\left(\mathrm{W}_{\mathrm{n}}\right)$ - number of weights of connection

- $(\mathrm{P})-$ Output

The nodes in the hidden and output layers are sometimes stated as processing units. The weighting vectors interconnect with the nodes of adjacent layers that are initially randomized, however, there are no connections between the nodes belonging to the same layer (Hou et al., 2008).

According to Payandeh et al. (2009), Processing Elements (PE) or neurons in the input layer only act as buffers for allocating the input signals, $X_{i}$ ( $i$ shows the $i$-th PE in the input layer) to PEs in the hidden layer. Each PE $j$ (j shows the $j$-th PE in the hidden layer and output layers) in the hidden layer calculates its input signals $X_{i}$ after weighing the values of the respective connections $W_{j i}$ from the input layer, then computes its output $Y_{j}$ as a function $f$ of the sum, viz.,

$Y_{j}=f\left(\sum W_{j i} X_{i}\right)$

There are many choices for the transfer function $\mathrm{f}$ which can be globally supported. The only limitation of this function is that it must be derivable.

Linear: $\mathrm{f}(\mathrm{x})=\mathrm{x}$

Log - Sigmoid: $\mathrm{f}(\mathrm{x})=\frac{1}{1+\mathrm{e}^{-\mathrm{x}}}$

Tan - Sigmoid: $\mathrm{f}(\mathrm{x})=\frac{2}{1+\mathrm{e}^{-2 \mathrm{x}}}-1$

Any supervised learning algorithm aims to find a function that best maps a set of inputs to its correct output. However, MLP comprises weight adjustments that are initially set by random values over a small range. Updating of weights continues until the output units of the 
net for each set of data inputs are as close as possible to the desired outputs. Mathematically speaking, the learning technique searches to minimize the following error function (Harchli et al., 2016):

$\mathrm{e}_{\mathrm{i}}=\mathrm{d}_{\mathrm{i}}-\mathrm{y}_{\mathrm{i}}$

Where $d_{i}$ is the desired response and $y_{i}$ is the calculated output by the network in response to the input $X_{i}$.

Multi-Layer Perceptron neural network uses a supervised learning technique called backpropagation (BP), which is commonly suggested as the most efficient procedure in the training of neural networks. This is used in conjunction with an optimization method such as gradient descent (Werbos, 1994). The Back Propagation (BP) method calculates the differences between the estimated and the measured values to define an error (Demuth and Beale, 2002). The error is then backpropagated through the network to update the weights and get the optimum results (Werbos, 1994). The mathematical expression of back-propagation ANN is represented by the following equations (Hou et al., 2008):

$$
\mathrm{x}=\sum \mathrm{W}_{\mathrm{i}} \mathrm{X}_{\mathrm{i}}
$$

$$
\mathrm{P}=\frac{2}{1+\mathrm{e}^{-\mathrm{x}}}-1
$$

Where $\mathrm{W}_{\mathrm{i}}$ is the weighting vector, $\mathrm{X}_{\mathrm{i}}$ is the input vector of neural network (seismic attribute), $\mathrm{x}$ is a weighting function, $\mathrm{P}$ is output (hydrocarbon and non-hydrocarbon) which is calculated by a sigmoid exponential function, and $\mathrm{n}$ is the number of input vectors (seismic attributes). The training algorithm attempts to minimize the error between the computed output and the desired output values by automatically adjusting the weighting vectors of connections. In other words, minimize the error on the test data by adjusting the weights related to all the interconnected perceptrons. When the errors fail to further reduce, the training is finished and the weighting vectors that yield the minimum error are saved, and the functions are determined (Hou et al., 2008).

\subsubsection{Implementation of multilayer perceptron (MLP) neural network architecture on the selected seismic attributes}

In figure 4, the neural network is a non-linear model with a description of the input seismic attributes (as shown on the left). Architecturally or topologically, this network consists of 5 nodes in the input layer, 12 nodes in the hidden layer, and 2 nodes in the output layer. Data is received through the nodes at the input layer, then the data is combined to activate the process with sigmoid activation function in nodes of hidden layer and results in either hydrocarbon (1) or non-hydrocarbon (0) in nodes of the output layer. The blue color code represents respective nodes with the synaptic weights given to each input. Though, these nodes are fully connected throughout the network. The predicted output (as shown on the right) indicates the presence of hydrocarbon (1) and the absence (0) of hydrocarbon respectively. The neural training was carried out iteratively. This updates the connection weights as well as overcoming problems related to local minima.

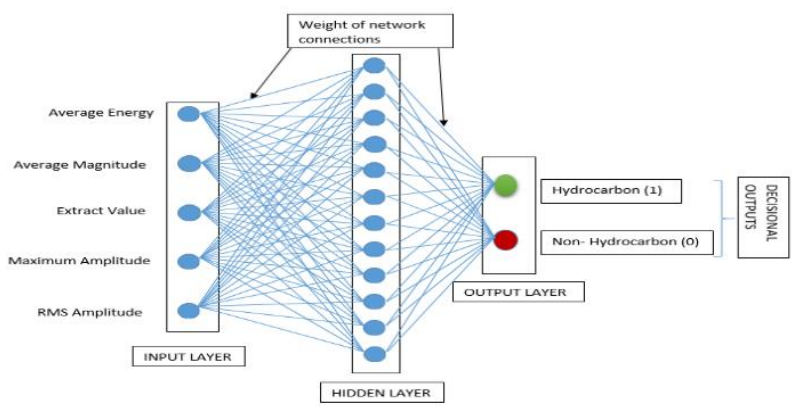

Fig. 4: Multilayer Perceptron Network (MLP) Architecture / Topology Used for Multi-Attribute Processing in the Study.

\section{Materials and methodology}

The research utilized post-stack three-dimensional seismic data, a suite of well log data (gamma-ray, resistivity, sonic, density, and neutron logs), checkshot data, Petrel 2015, Microsoft Excel 2016, Microsoft Command Prompt line 10.0.11362.243, and Python 3.47. The well logs were used for formation evaluation. A synthetic seismogram was generated from the check shot data for a well-to-seismic tie. The seismic data was interpreted structurally by picking faults based on abrupt termination, change in pattern, and dip of events. The checkshot data was used for time to depth conversion of the observed time structural map. The individual seismic attributes were generated and exported as numerical values for machine learning analysis. As part of the machine learning analysis, importation of relevant Python libraries e.g Numpy, Panda, Matplotlib, SimpSom, and Keras were done using the Microsoft Command Prompt line 10.0.11362.243. In addition, data exploration and transformation processes were conducted on exported seismic attribute data using Microsoft Excel 2016. A computer programme (in Python language) was developed to combine relevant seismic attributes for the generation and performance assessment of hydrocarbon predictive models. For supervised Multi-Layer Perceptron Neural Network (MLPNN) predictive models, the seismic attributes dataset was automatically shuffled and split up into 70\% train and 30\% test data set. Then, it was fitted into the MultiLayer Perceptron (MLP) machine learning algorithm for data training, testing, and prediction. Also, the seismic attributes dataset was directly fitted into the unsupervised Self- Organizing Map (SOM) machine learning algorithm. This was used for data training, clustering, prediction, and visualization. Comparative performance analysis of the machine-assisted predictive models (Multi-Layer Perceptron and Self- Organizing Map Neural Network) was conducted based on Binary Cross-Entropy classification metric/ visual inspection. The best result from comparative performance analysis was also validated using the single-generated seismic attribute (Maximum Amplitude and Extract Value) map. A correlation of well depth-to-top of selected horizons with the seismic depth sliced surface was used for further model validation.

Figure 5 shows the methodology flowchart adopted for the supervised Multi-Layer Perceptron (MLP) machine learning open-source codes. There are five (5) stages: Input patterns, parameter setting, MLP Training, MLP model evaluation, and epoch/iteration. At the input patterns stage, the seismic attribute data was loaded and automatically split into the training and testing dataset. At the parameter setting stage, the 
following were supplied: selection of network, activation function, number of the epoch, and batch size. At the MLP training process, for each batch size of the epoch, the training (seismic attribute) data from the input pattern stage was passed through the parameter setting and MLP training process to produce a predictive output $(0 \& 1)$. There is a feed-forward of weighted connections between the neural network nodes. The error is defined by calculating the differences between estimated and measured values across the neural network nodes (Demuth and Beale, 2002). This error is stored and then backpropagated through the network to update the weights and get the optimum result (Werbos, 1994). At the MLP model evaluation stage, the prediction result (model) was evaluated with the testing (seismic attribute) data, using a classification performance metric called "Binary Cross Entropy". This was based on its accuracy and precision. At the epoch/ iteration stage, these processes continue till the end of the specified iteration (epoch) number.

Figure 6 shows the methodology flowchart adopted for the unsupervised Self- Organizing Map (SOM) machine learning open-source codes. There are four (4) stages: Input patterns, parameter setting, SOM processes, and epoch/iteration. At the input patterns stage, the seismic attribute data were loaded as input patterns. At the parameter setting stage, the following was provided: network dimension, number of epochs, and learning rate. At the SOM process, for each learning rate of the epoch, the input pattern passes through the SOM processes (competition, cooperation, and synaptic adaption) to give a weight update at the output layer. After the initialization of the SOM processes with some set of values. This resulted in the neurons competing with each other to identify the winner neuron - Competition. This winning neuron determines the spatial location of a topological neighborhood of excited neurons - Cooperation (Al Moqbel and Wang, 2011). An adjustment is applied to the synaptic weight of the excited neurons - Synaptic Adaption (Burguillo and Dorronsoro, 2013). At the epoch/ iteration stage, these processes continue till the end of the specified iteration (epoch) number (Roden et al., 2015).

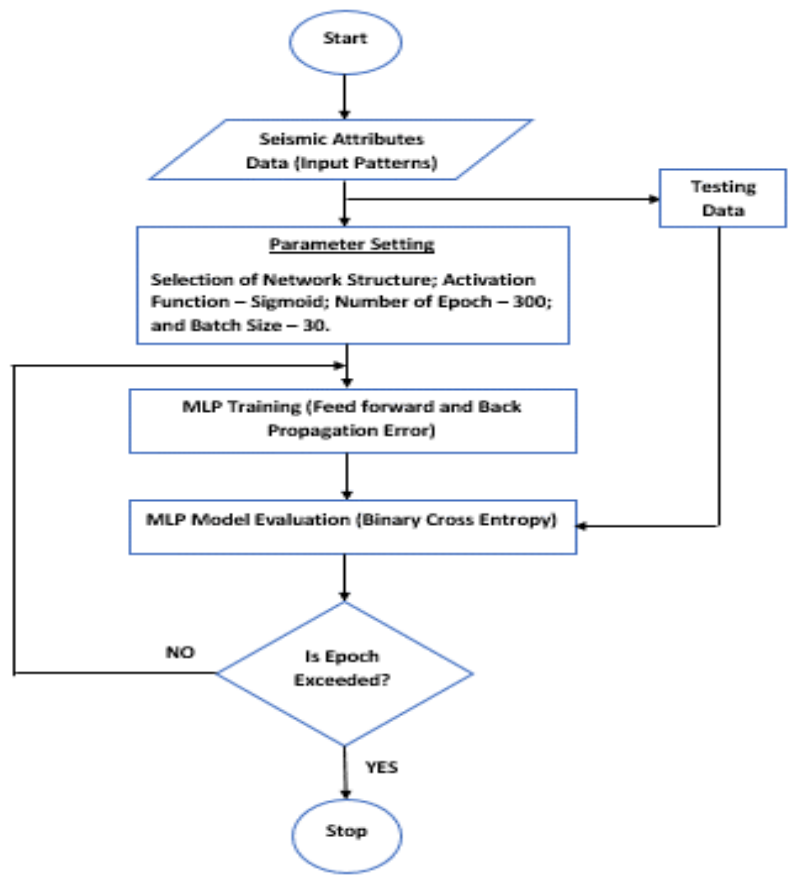

Fig. 5: Methodology Flowchart Adopted for the Supervised Multi-Layer Perceptron (MLP) Machine Learning Open-Source Codes.

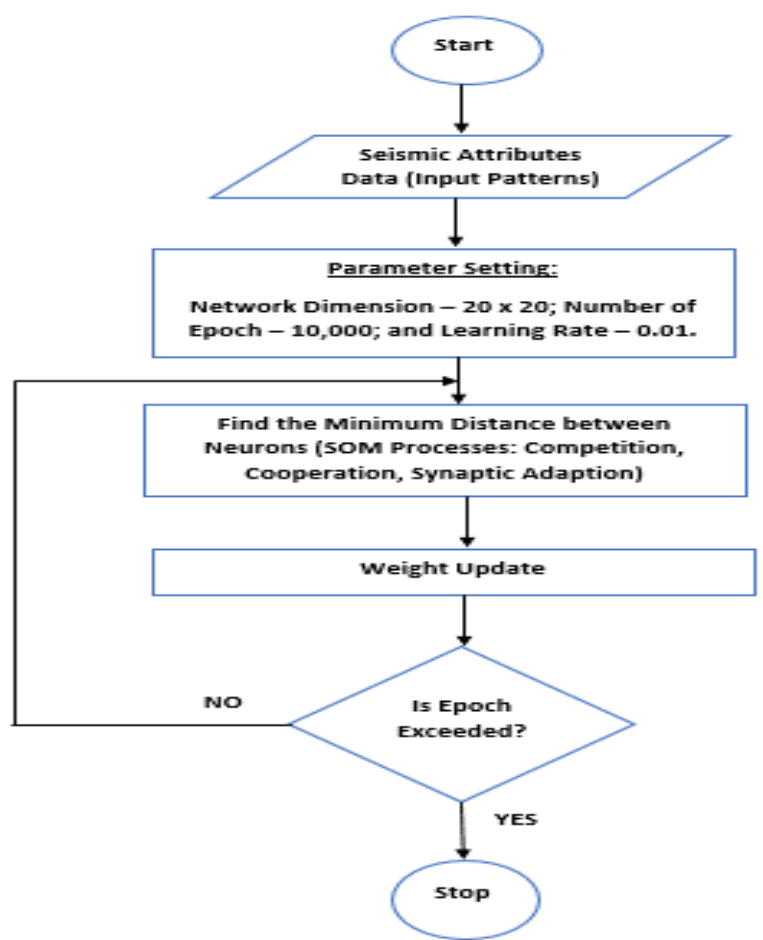

Fig. 6: Methodology Flowchart Adopted for the Unsupervised Self- Organizing Map (SOM) Machine Learning Open-Source Codes. 


\section{Results and discussion}

\subsection{Structural interpretation}

Figure 7 is a typical seismic section (Inline 11419) that shows the mapped faults and horizons for the structural geometry of the "GEM" Field. On this seismic profile, there are three mapped horizons - Horizon1, Horizon2, and Horizon3 - corresponding to the tops of the identified reservoirs across the field. In addition, there is clear evidence of faulting activities that have affected the rocks in the study area. A total of six (6) faults were delineated (Fault1 - Fault6). They include three antithetic (Fault3, Fault5, and Fault6) faults trending in the Southwest - Northeastern direction and one synthetic fault (Fault4) trending in the North West- South East direction. Thus, they can serve as entrapment of the migrated hydrocarbon in the study area.

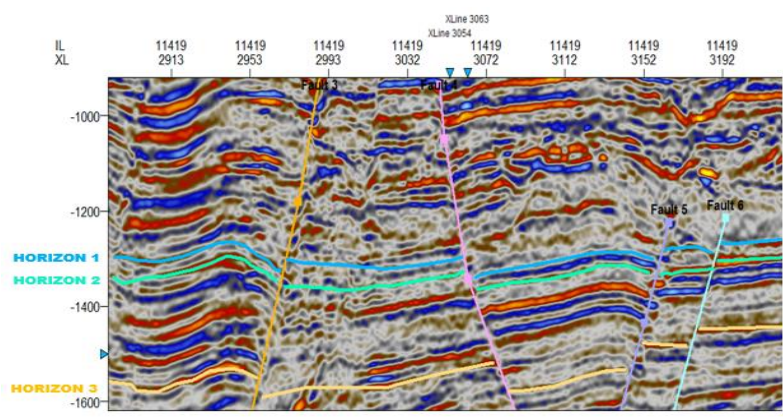

Fig. 7: Inline 11419 Showing the Mapped Faults and Horizons.

Figure 8 shows a depth structural map for Horizon 1 spanning over the depth range of $4150-4950 \mathrm{ft}$. This map was derived from the isochron ma, not shown, using checkshot data. It depicts the depth configuration across the seismic horizon. The high depth values (deep blue to purple colours) indicate synclinal structures whereas the low depth values (yellow to green colours) indicate anticlinal structures. The structural map shows an NW-SE trending anticlinal structure with its peak at the north-central part of the field. Well GEM 04, 21, 05, 32 , and 38 were suspected to be rightly located to target the fault (F2 and F3)-assisted closure, favourable for hydrocarbon accumulation.

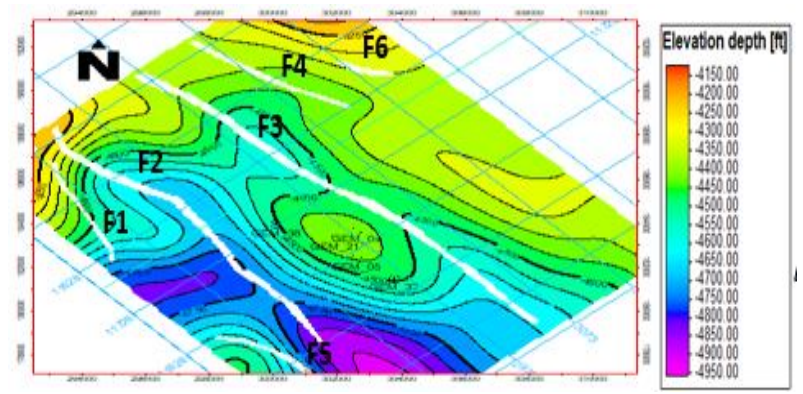

Fig. 8: Depth Structural Map of Horizon 1.

\subsection{Seismic attribute analysis}

Figures 9, 10, 11, 12, and 13 show five seismic attribute maps (RMS amplitude, Extract value, Maximum Amplitude, Average Energy, and Average magnitude) for hydrocarbon detection analysis. The attribute maps revealed the amplitude of seismic reflection across the mapped horizons. These are due to the observed acoustic impedance contrast anomaly. The high amplitude zones (around the NW-SE area) were regarded as hydrocarbon zone due to high acoustic impedance whereas low amplitude areas indicate the non-hydrocarbon due to low acoustic impedance contrast. A comparative analysis of the features of the attribute maps and the structure map. This revealed a good relationship among the maps with the structural high areas (anticlines) coinciding with the bright spot areas (acoustic impedance contrast) observed on the attribute maps. Furthermore, a comparative analysis of the generated five seismic attribute maps revealed that there is a bit of inconsistent amplitude variation of the same area indicating hydrocarbon presence. So, interpreting based on a single-generated seismic attribute wouldn't image direct optimal hydrocarbon presence and leads to the drilling of a dry hole. At this point, machine learning as a veritable tool is used to combine relevant attributes for enhancing/ improving the visibility of those areas as well as predicting other areas of hydrocarbon presence.

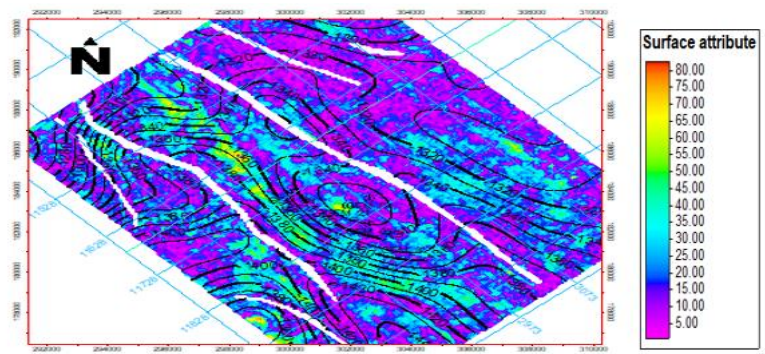

Fig. 9: RMS Amplitude Seismic Attribute map. 


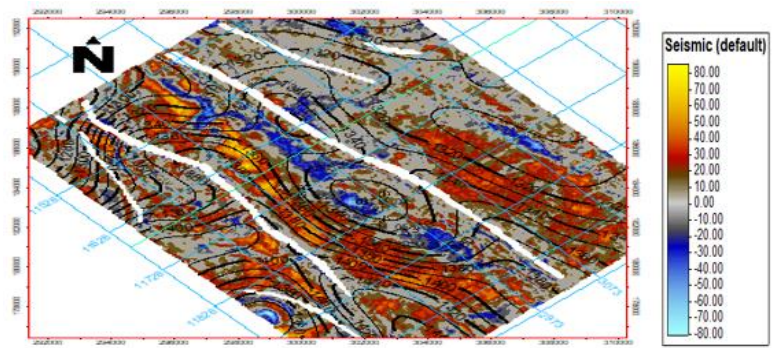

Fig. 10: Extract Value Seismic Attribute Map.

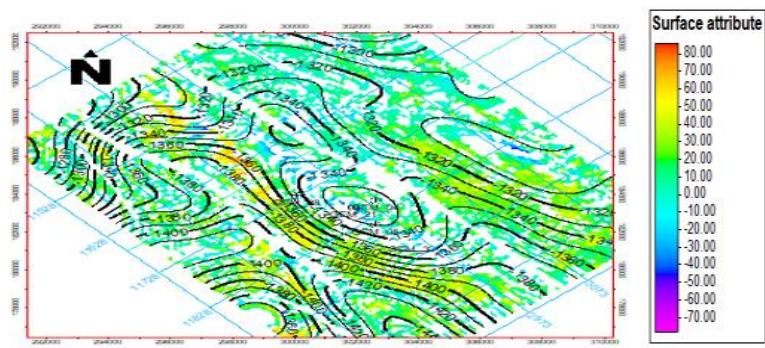

Fig. 11: Maximum Amplitude Seismic Attribute Map.

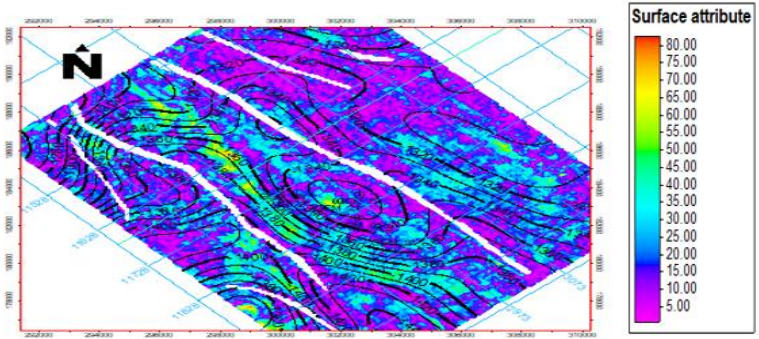

Fig. 12: Average Magnitude Seismic Attribute Map.

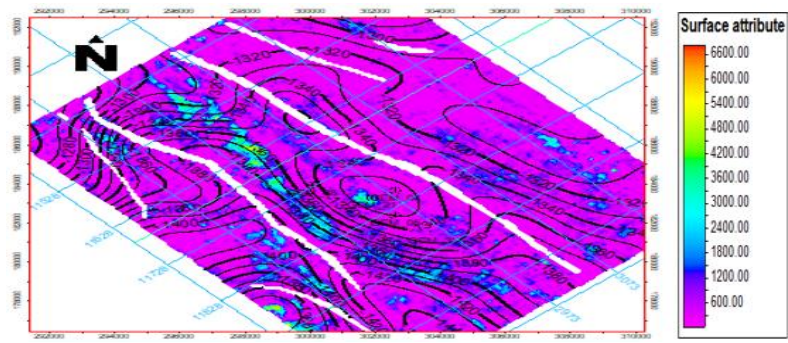

Fig. 13: Average Energy Seismic Attribute Map.

\subsection{Machine learning analysis}

The machine learning analysis provides an automated system for learning and improvement from input data (seismic attributes) which predict hydrocarbon zone in the "GEM" field. The machine learning analysis entails data exploration analysis, development, and performance evaluation of the predictive models.

\subsubsection{Data exploration analysis: seismic attribute histogram}

Figure 14 shows five seismic attribute histograms (RMS amplitude, Maximum Amplitude, Average Energy, Average magnitude, and Extract value) were generated from the extracted seismic attribute maps. These seismic attribute histograms were used to gain a better understanding of the patterns/trends and distribution of each seismic attribute data point through data visualization. These plots show the percentage frequency occurrence of amplitude value in the seismic attribute data point distributions. These have been divided into the number of class intervals called bins. These bins have a range of values that is proportional to a given percentage frequency occurrence of amplitude value in the seismic attribute data point distributions. The longest bins indicate the highest percentage frequency occurrence of amplitude values whereas the shortest bins indicate otherwise. Most of the seismic attribute histograms are skewed right. Thus, this indicates the mean of the seismic attribute dataset is greater than the median. Table 1 shows the amplitude pattern types and their corresponding frequency occurrence in seismic data point distributions. The observed amplitude pattern types were grouped into high, moderately high, moderately low, and low for each seismic attribute. These were grouped with their corresponding seismic attribute histogram indicating frequency occurrence in seismic data point distributions.

Table 1: Amplitude Pattern Types and its Corresponding Frequency Occurrence in Seismic Datapoint Distributions

\begin{tabular}{|c|c|c|c|c|c|}
\hline Seismic Attribute Name & Amplit & ern Types and Cor & equency Occurrer & & \\
\hline & High & Moderately high & Moderately low & Low & Total \\
\hline Average Energy & $38.5 \%$ & $24.2 \%$ & $30.5 \%$ & $6.7 \%$ & $100 \%$ \\
\hline Average Magnitude & $40.4 \%$ & $29.1 \%$ & $17.4 \%$ & $13.1 \%$ & $100 \%$ \\
\hline Extract Value & $39.0 \%$ & $30.6 \%$ & $17.9 \%$ & $4.9 \%$ & $100 \%$ \\
\hline
\end{tabular}




\begin{tabular}{|c|c|c|c|c|c|}
\hline Maximum Amplitude & $46.9 \%$ & $36.2 \%$ & $9.9 \%$ & $7.1 \%$ & $100 \%$ \\
\hline RMS Amplitude & $40.4 \%$ & $29.1 \%$ & $17.4 \%$ & $13.1 \%$ & $100 \%$ \\
\hline
\end{tabular}

(A)

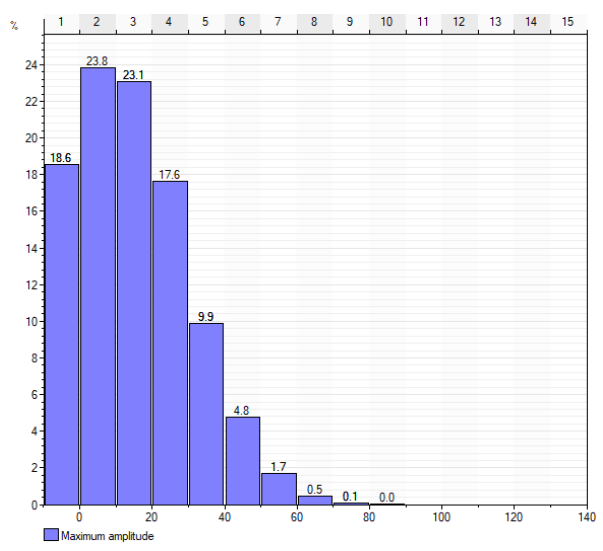

(C)

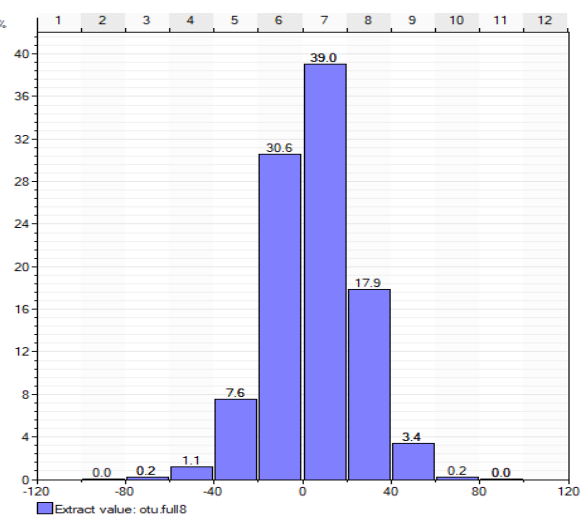

(B)

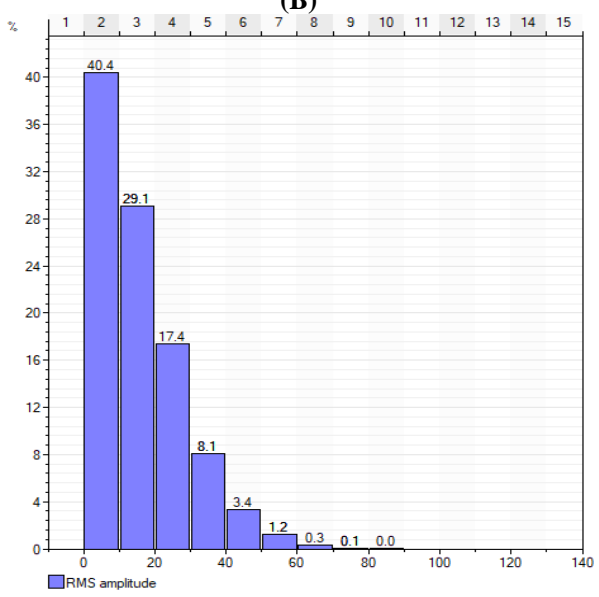

(D)

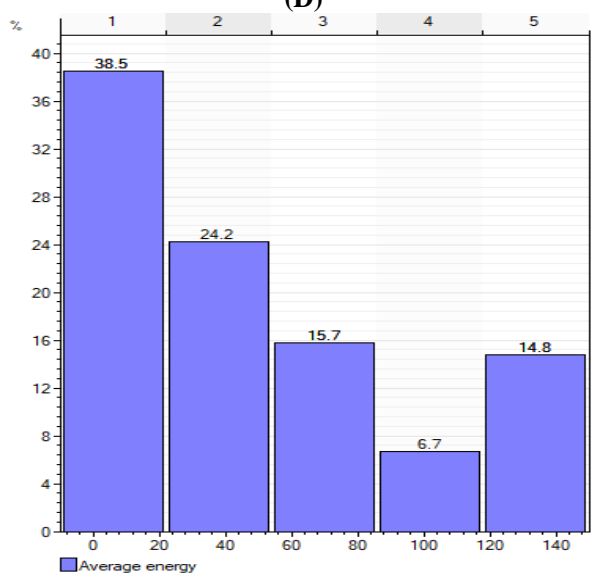

$(\mathbf{E})$

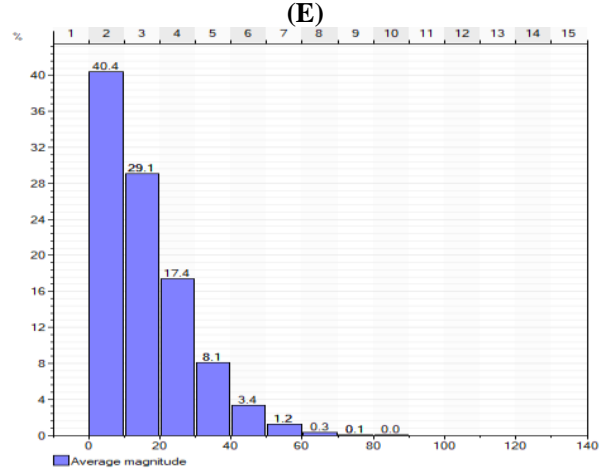

Fig. 14: Seismic Attribute Histogram Showing: (A) Maximum Amplitude, (B) RMS Amplitude, (C) Extract Value (D) Average Energy (E) Average Magnitude.

\subsubsection{Development and performance evaluation of multi-layer perceptron (MLP) neural network predictive model}

Figure 15 shows the MLP predictive model map used to represent the zone of predicted hydrocarbon and non-hydrocarbon in the "GEM" Field. The green spots observed in this map indicate the predicted hydrocarbon zones whereas the red spots indicate the predicted nonhydrocarbon. The green areas were observed within and around fault-assisted closure (NW-SE region) which possibly confirmed the location choice of drilling wells within the study area. However, the predictive model performance was evaluated using a classification metric called "binary cross-entropy". It was observed that after several iterations, the overall binary cross-entropy measured the model accuracy and loss for the train and test data over a specific epoch. Moreover, the train and test learning curves converged to a stabilized value with a minimal gap between two final values over a specific epoch. Therefore, this indicates the machine learning algorithm learnt from the given training dataset and formed a good fit. For the model accuracy, it attained a training variation of $0.98-0.99$ and a testing variation of $0.96-0.989$ over 140 epochs. The measured values tend towards 1 . Thus, this indicates a high degree of predictive model accuracy. On the other hand, model loss attained a training variation of $0.03-0.05$ and a testing variation of $0.08-0.12$ over the same epoch. The measured values tend towards 0 . Hence, it indicates a good probability prediction between ( 1 and 0 i.e., hydrocarbon presence or not in a zone) with minimal error occurrence (Figure $15 \mathrm{a}$ and $\mathrm{b}$ ). 

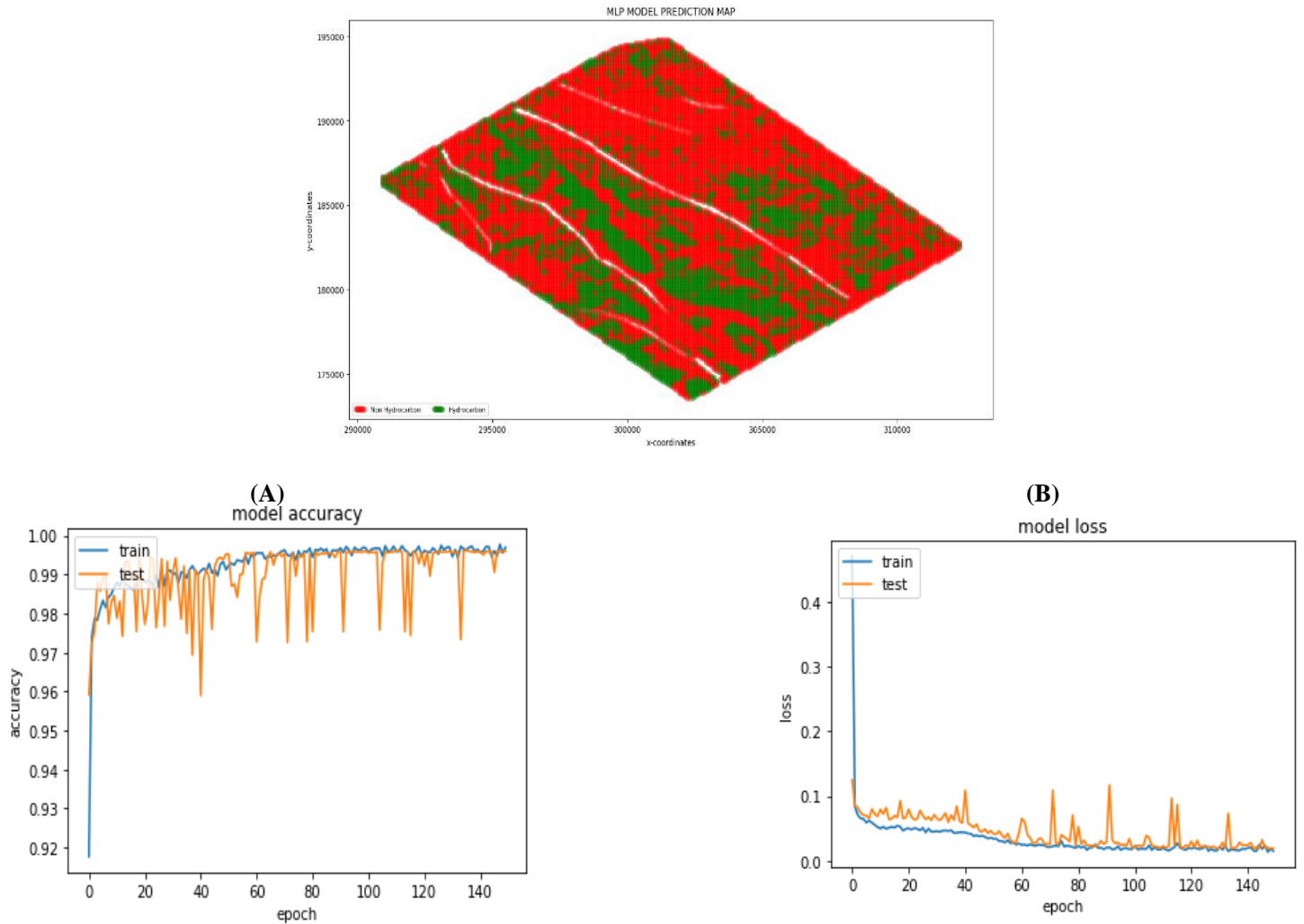

Fig. 15: Multi-Layer Perceptron Model Map and Its Performance Evaluation for Hydrocarbon Prediction; Based on (A) Model Accuracy and (B) Model Loss.

\subsubsection{Development of self-organizing map neural network predictive model}

Figure 16 shows the neural topology/architecture of 400 neurons (20x20) for clustering and predicting the zone of hydrocarbon presence. This was used along with the following five attributes: RMS amplitude, Maximum Amplitude, Average Energy, Average magnitude, and Extract value. The SOM analysis identifies and clusters from different combinations of seismic attributes. This reveals significant information about the classification structure of natural groups in an associated 2D colormap. A close visual inspection of the SOM results shows the $2 \mathrm{D}$ colormap is a correlation of colors to neurons which ranges from deep blue - yellow. Deep blue neurons/ nodes indicating non - hydrocarbon and yellow neurons indicate hydrocarbon as a result of seismic amplitude values in it. The hydrocarbon clustered neurons are situated in the central part of the 2D color map whereas the non-hydrocarbon clustered neurons are evenly distributed on the map. Furthermore, a close visual inspection of the Self-Organizing Map (SOM) results showed that the after-training 2D color map learnt/mimic to a good extent when compared to the initialized 2D color map. Each winning neuron with the largest amplitude value is surrounded by similar input seismic amplitude values. The deep blue neuron/ node amplitude values range from $0-200$ whereas yellow neuron amplitude values range from 1200 - 1300 after the training/ clustering process at 1000 epoch.

(A)

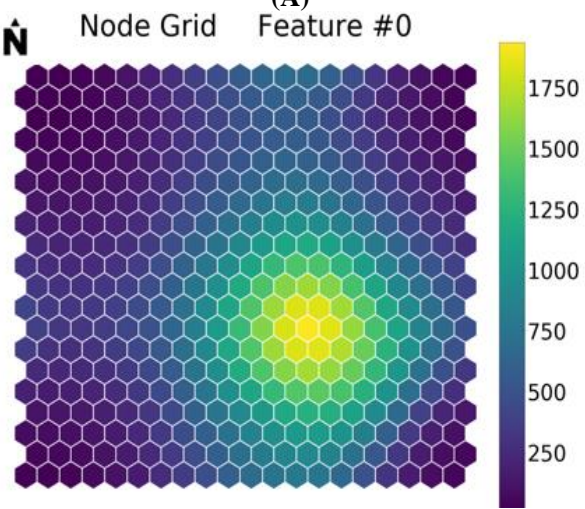

(B)

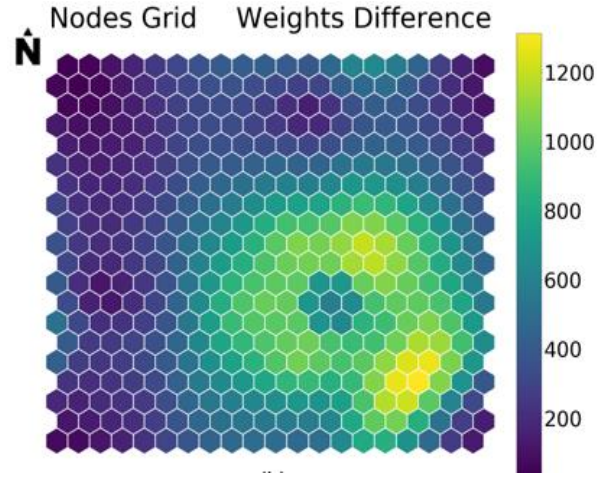

Fig.16: SOM Hydrocarbon Predictive Model; (A) Initialization / Visualization Process (B) After Training/ Clustering Process.

\subsubsection{Model validation: hydrocarbon prospect}

Figure 17 shows the correlation of MLP model prediction map, Maximum Amplitude, and Extract Value for model validation and hydrocarbon prospect in the study area. The purple circle was used to spot similar positions with hydrocarbon presence across the compared maps whereas a blue rectangle was used to spot the enhanced visibility of hydrocarbon zones across the same maps. The Multi-layer Perceptron (MLP) model predicted hydrocarbons with a training accuracy variation of $0.98-0.99$ as well as a testing accuracy variation of $0.96-0.989$ over their epoch interval (Figure 15). Six (6) hydrocarbon prediction zones were identified on the MLP predictive model map. This conformed to the individual seismic attribute (Maximum amplitude and Extract value) maps indicating high amplitude (hydrocarbon presence). In addition, the MLP model results enhanced the visibility of the other five (5) hydrocarbon prediction zones. This captured the minute details of combined relevant seismic attributes as compared to the individual seismic attributes. Therefore, this 
increases the confidence level of efficient well placement as well as reservoir perforation using MLP predictive model map over SelfOrganising Map (SOM) predictive model.

Table 2 shows a correlation of well depth-to-top of selected horizons with the seismic depth slice surface. This was used for further model validation. The depth slice remains constant at each horizon and has a variation of $4400 \mathrm{ft}, 4800 \mathrm{ft}$, and $5600 \mathrm{ft}$ at their respective horizon. The well depth to top values increases with the mapped horizons as deduced from well logs. These selected well locations (GEM 05, 38, and 32) are closer to zones that are indicative of hydrocarbon presence on seismic attribute maps and MLP predictive model maps. The percentage depth deviation has a varied range of $\pm 0.5 \%- \pm 16.7 \%$ across the three mapped horizons. Thus, it determines the success rate of hydrocarbon presence with minimal errors. These minimal errors can serve as a possible measure for efficient well placement of the other five (5) hydrocarbon prediction zones.

Table 2: A Correlation of Well Depth-to-Top of Selected Horizons with the Seismic Depth Slice Surface

\begin{tabular}{llllll}
\hline \multicolumn{5}{c}{ Tabiz 2: } \\
\hline \multirow{4}{*}{1} & Well Name & Well Depth to Top (ft) & Depth Slice (ft) & Depth Deviation (ft) & \% Depth Deviation \\
\hline & GEM 05 & 4420 & 4400 & 20 & $\pm 0.5 \%$ \\
& GEM 38 & 4900 & 4400 & 500 & $\pm 10.2 \%$ \\
2 & GEM 32 & 4540 & 4400 & 140 & $\pm 3.1 \%$ \\
& GEM 05 & 4550 & 4800 & -250 & $\pm 5.6 \%$ \\
& GEM 38 & 5100 & 4800 & 300 & $\pm 5.9 \%$ \\
& GEM 32 & 4700 & 4800 & -100 & $\pm 2.1 \%$ \\
3 & GEM 05 & 4800 & 5600 & -800 & $\pm 16.7 \%$ \\
& GEM 38 & 5450 & 5600 & -150 & $\pm 2.8 \%$ \\
& GEM 32 & 4925 & 5600 & -675 & $\pm 13.7 \%$ \\
\hline
\end{tabular}

(A)

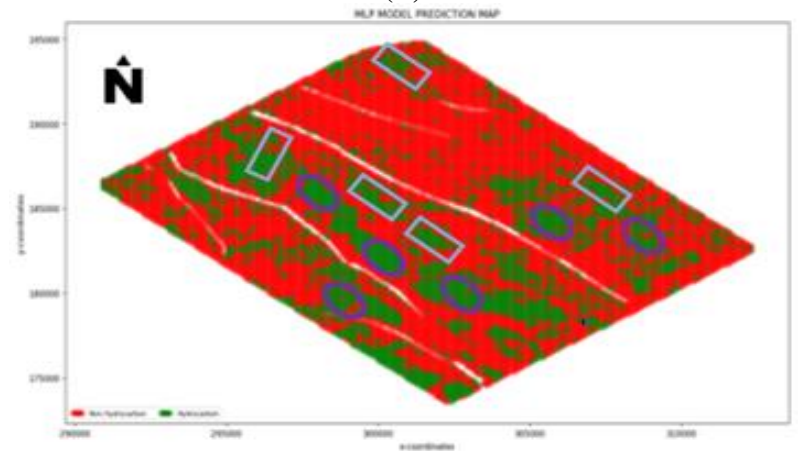

(B)

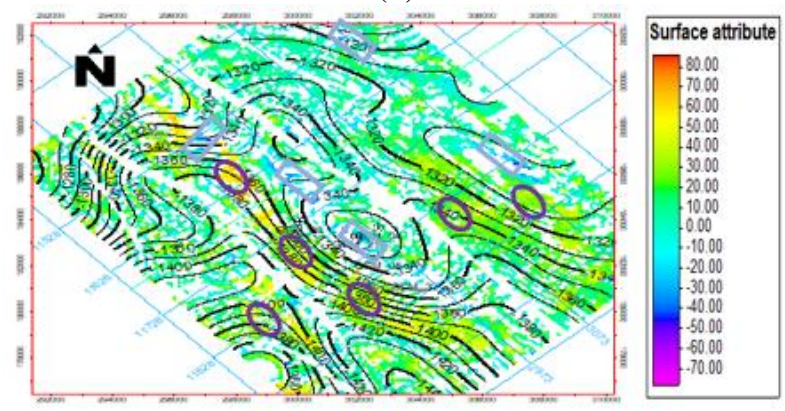

(C)

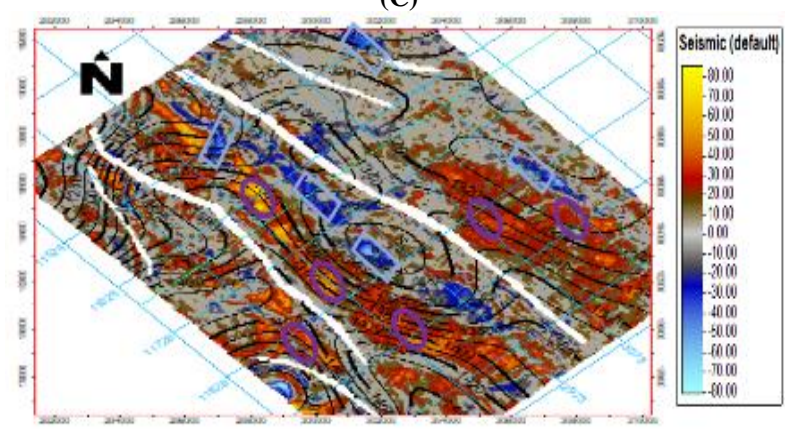

Fig. 17: Correlation of (A) MLP Model Prediction Map, (B) Maximum Amplitude, and (C) Extract Value for Hydrocarbon Prospect.
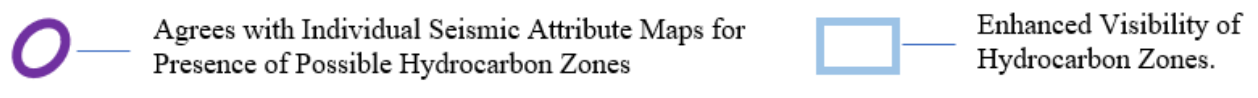

\section{Conclusion}

The study has attempted to delineate subsurface geologic structures favourable for hydrocarbon accumulation using surface seismic and well log data. In all, six faults (Fault1 - Fault6) and three (3) depth surfaces were mapped across the field. The seismic attribute analysis 
revealed hydrocarbon zones around the delineated structures and new prospects. The Multi-Layer Perceptron (MLP) and Self-Organizing Map (SOM) Neural Network algorithm were employed to combine five (5) relevant seismic attributes for predicting hydrocarbon zones. The python codes were developed for the generation and performance assessment of predictive models. The assessment of the developed MLP predictive models' performance was based on training and testing accuracy of $0.96-0.99$ over 140 epochs for hydrocarbon prediction zones. Thus, this indicates a high degree of predictive model accuracy and formed a good fit. Overall, the Multi-Layer Perceptron (MLP) predictive model map gave higher precision of the predicted hydrocarbon zones over the Self- Organizing Map (SOM) predictive model map, as a result of its supervised nature, thus reinforcing the confidence level of using the former. On model validation, six (6) hydrocarbon prediction zones were identified on the MLP predictive model map. This conformed to the individual seismic attribute (Maximum amplitude and Extract value) maps indicating high amplitude (hydrocarbon presence). In addition, the MLP model results enhanced the visibility of the other five (5) hydrocarbon prediction zones. A correlation of well depth-to-top of selected horizons with the seismic depth slice surface was used for further model validation. The percentage (\%) depth deviations were mostly below $\pm 10 \%$. Therefore, this lends credence to the applicability and reliability of the employed machine learning algorithm for the prediction of hydrocarbon zones in the study area.

\section{Additional information}

Data related to this research has been kept online at: https://github.com/fredrickoshogbunu/Machine-Learning-Approach-to-HydrocarbonPrediction-from-Seismic-Attributes

\section{References}

[1] Al Moqbel, A., and Wang, Y. (2011). Carbonate Reservoir Characterization with Lithofacies Clustering and Porosity Prediction. Journal of Geophysics and Engineering, vol. 8, pp. 592-598 https://doi.org/10.1088/1742-2132/8/4/011.

[2] Burguillo, J.C. and Dorronsoro, B. (2013). Using Complex Network Topologies and Self- Organizing Maps for Time Series Prediction. Advances in Intelligent Systems and Computing.p1-10. https://doi.org/10.1007/978-3-319-00542-3_33.

[3] Chehrazi, A., Rahimpour-Bonab1, H. and. Rezaee, M.R. (2013). Seismic Data Conditioning and Neural Network-Based Attribute Selection for Enhanced Fault Detection. Petroleum Geoscience, Vol. 19, 169-183, https://doi.org/10.1144/petgeo2011-001.

[4] Chopra, S., and Marfurt, K. (2007). Seismic Attributes for Prospect Identification and Reservoir Characterization. SEG Geophysical Development Series. https://doi.org/10.1190/1.9781560801900.

[5] Demuth, H., Beale, M. (2002). Neural Network Toolbox for Use with MATLAB, User's Guide, Version 4. The Math Work, Inc, Natick.

[6] Harchli, F., Joudar, N., Es-Safi, A. and Ettaouil, M. (2016). Adaptation of Multilayer Perceptron Neural Network to Unsupervised Clustering using a Developed Version of the K-means Algorithm. WSEAS Transactions on Computers. 15, p103- 116.

[7] Haykin, S. (2009). Neural Networks and Learning Machines. 3rd ed. New Jersey, USA: Pearson Prentice Hall. p. 1-894

[8] Hou, J., Takakashi, T., Katoh, A., Jaroonsithaz, S., Chumsenaz, P.K. and Nakayama, K. (2008). Application of seismic attributes and Neural Network for sand probability prediction-A case study in the North Malay Basin. Bulletin of the Geological Society of Malaysia. 54, p. 115 - 121 https://doi.org/10.7186/bgsm54200818.

[9] Hurwitz, J. and Kirsch, D. (2018). Machine Learning for Dummies. New Jersey, USA: John Wiley \& Sons, Inc. p1-67

[10] Kohonen, T. (1982). Self-Organized Formation of Topologically Correct Feature Maps: Biological Cybernetics, 43, 59-69, https://doi.org/10.1007/BF00337288.

[11] Kumar, P. C. and Mandal, A. (2018). Enhancement of Fault Interpretation Using Multi-Attribute Analysis and Artificial Neural Network (ANN) Approach: A Case Study from Taranaki Basin, New Zealand. Exploration Geophysics, 49, 409-424, https://doi.org/10.1071/EG16072.

[12] Payandeh, M., Mehrnoush Aeinfar, M., Aeinfar, V., and Hayati, M. (2009). A New Method for Diagnosis and Predicting Blood Disorder and Cancer Using Artificial Intelligence. International Journal of Hematology-Oncology and Stem Cell Research. 3 (4), p25-33.

[13] Ralhan, A. (2018). Self-Organizing Maps. [ONLINE] Available at: https://towardsdatascience.com/self-organizing-maps-ff5853a118d4. [Accessed 20th Jun 2019].

[14] Roden, R. and Chen, C. (2017). Interpretation of DHI Characteristics with Machine Learning. First Break, 35, 55-63. https://doi.org/10.3997/13652397.35.5.88069.

[15] Roden, R., Smith, T., Santogrossi, P., Sacrey, D. and Jones, G. (2017). Seismic Interpretation Below Tuning with Multi- Attribute Analysis. The Leading Edge, 36, 330-339. https://doi.org/10.1190/tle36040330.1.

[16] Santogrossi, P. (2017). Technology Reveals Eagle Ford Insights. American Oil \& Gas Reporter, January.

[17] Sacrey, D. and Roden, R. (2018). Solving Exploration Problems with Machine Learning. First Break, 36, 67-72. https://doi.org/10.3997/13652397.n0100.

[18] Shalev-Shwartz, S and Ben-David, S. (2014). Understanding Machine Learning: From Theory to Algorithms. New York, USA: Cambridge University Press. p1- 499.

[19] Singh, S. (2018). Cousins of Artificial Intelligence. [ONLINE] Available: https://towardsdatascience.com/cousins-of-artificial-intelligencedda4edc27b55 [Accessed 5th Feb 2019].

[20] Singh Gill, N. (2019). Artificial Neural Networks and Neural Networks Applications. [ONLINE] Available: https://www.xenonstack.com/blog/artificial-neural-network-applications/. Last accessed 20th Jun 2019.

[21] Smith, T. and Taner, M.T. (2010). Natural Clusters in Multi-Attribute Seismic Found with Self-Organizing Maps. Extended Abstracts, RobinsonTreitel Spring Symposium by GSH/SEG, March 10-11, 2010, Houston, Texas.

[22] Werbos, P.J. (1994). The Roots of Backpropagation. From Ordered Derivatives to Neural Networks and Political Forecasting. New York, USA: John Wiley \& Sons. p. 1-319.

[23] Willems, K. (2019). Keras Tutorial: Deep Learning in Python. Available: https://www.datacamp.com/community/tutorials/deep-learning-python. [Accessed 20th Jun 2019].

[24] Zhao, T., Li F., and Marfurt, K. J. (2016). Advanced Self-Organizing Map Facies Analysis with Stratigraphic Constraint. 86th Annual International Meeting, SEG, Expanded Abstract, 1666- 1670 https://doi.org/10.1190/segam2016-13949728.1.

[25] Zhao, T., Li, F., and Marfurt, K.J. (2017). Constraining Self-Organizing Map Facies Analysis with Stratigraphy: An Approach to Increase the Credibility in Automatic Seismic Facies Classification. Interpretation, 5, 2, p. T163-T171, https://doi.org/10.1190/INT-2016-0132.1.

[26] Zhao, T., Jayaram, V., Roy, A., and Marfurt, K. J. (2015). A Comparison of Classification Techniques for Seismic Facies Recognition. Interpretation, 3, no. 4, SAE29-SAE58, https://doi.org/10.1190/INT-2015-0044.1. 